\title{
La educación científica rural en la modalidad m-learning y su afectación en la pandemia de la covid-19
}

\author{
Carlos Enrique Rodríguez-Sarmiento ${ }^{1}$ \\ ${ }^{1}$ Universidad de Santander (UDES), Colombia
}

Resumen. Desde un aporte científico, social y educativo, este artículo muestra el proceso de enseñanzaaprendizaje de la ciencia física con el modelo m-learning, además, reflexiona sobre las repercusiones que conlleva la aplicación del modelo educativo durante la pandemia en los estudiantes de secundaria de un colegio público rural de Colombia en dos facetas.

En la primera faceta se evidencia que con el m-learning desde un enfoque constructivista, se está llevando la física con efectividad al lugar donde están los estudiantes, contribuyendo con su posibilidad de aislamiento social a que la covid-19 sea contenida. En la ruralidad, el teléfono móvil inteligente y la aplicación WhatsApp se destacan por su portabilidad y ventajas económicas.

En la segunda faceta mediante una encuesta virtual se aplica un instrumento de cuatro escalas que miden el ambiente familiar, el ciberacoso, la satisfacción del estudiante y la disposición hacia el estudio. Se encuentra que la disposición para el estudio es explicada por el clima familiar (24\%) y por la satisfacción del servicio educativo (51\%). La evidencia sugiere que la población estudiantil está siendo afectada con el m-learning en la pandemia repercutiendo en la disposición hacia el estudio, por lo que la educación en el sector rural estaría involucionando.

Palabras clave: aprendizaje móvil; covid-19; ambiente familiar; ciberacoso; satisfacción del estudiante; disposición hacia el estudio.

A educação científica rural na modalidade de m-learning e o seu impacto na pandemia covid-19

Resumo. A partir de uma contribuição científica, social e educacional, este artigo mostra o processo ensino-aprendizagem das ciências físicas com o modelo M-Learning, e reflecte também sobre as repercussões da aplicação do modelo educacional durante a pandemia em estudantes do ensino secundário numa escola pública rural na Colômbia, em duas facetas.

A primeira faceta mostra que o m-learning, a partir de uma abordagem construtivista, está efectivamente a levar a física para o local onde os estudantes se encontram, contribuindo com a sua possibilidade de isolamento social para a contenção da covid-19. Nas zonas rurais, o smartphone e a aplicação WhatsApp destacam-se pela sua portabilidade e vantagens económicas.

Na segunda faceta, através de um inquérito virtual, é aplicado um instrumento com quatro escalas que mede o ambiente familiar, cyberbullying, satisfação dos estudantes e vontade de estudar. A vontade de estudar é explicada pelo clima familiar (24\%) e pela satisfação com o serviço educativo (51\%). As evidências sugerem que a população estudantil está a ser afectada pela m-learning na pandemia, afectando a vontade de estudar, de modo que a educação no sector rural seria involuntária.

Palavras-chave: aprendizagem móvel; covid-19; ambiente familiar; cyberbullying; satisfação do estudante; vontade de estudar.

Rural science education in the m-learning modality and its impact on the covid-19 pandemic Abstract. From a scientific, social and educational contribution, this article shows the teaching-learning process of physical science with the m-learning model, in addition, it reflects on the repercussions of the application of the educational model during the pandemic in high school students of a rural public school in Colombia in two facets.

In the first facet it is evidenced that with m-learning from a constructivist approach, physics is being effectively taken to the place where the students are, contributing with its possibility of social isolation to Covid-19 being contained. In rurality, the smart cell phone and the WhatsApp application stand out for their portability and economic advantages.

In the second facet by means of a virtual survey, an instrument of four scales measuring family environment, cyberbullying, student satisfaction and disposition towards study is applied. It is found that willingness to study is explained by family climate (24\%) and satisfaction with the educational service (51\%). The evidence suggests that the student population is being affected by m-learning in the pandemic, affecting the willingness to study, so that education in the rural sector would be involuted. Keywords: m-learning; covid-19; family environment; cyberbullying; student satisfaction; disposition toward study. 


\section{Introducción}

El trabajo aborda un tema educativo de interés y actualidad, el aprendizaje móvil (m-learning) para la enseñanza de la Física en una zona rural del departamento de Santander - Colombia, a cuyo alumnado se debe llegar a través de los teléfonos móviles debido al cierre de la enseñanza presencial derivado de la situación de pandemia por la covid-19. Para desarrollar esos procesos de enseñanza-aprendizaje se implementan secuencias didácticas orientadas a través de WhatsApp, analizando, además, en qué medida el alumnado se siente motivado/comprometido con su aprendizaje en estas circunstancias.

El problema consiste en la necesidad de implementar con efectividad el Aprendizaje Móvil como estrategia educativa para desarrollar el proceso de enseñanzaaprendizaje de la ciencia física en casa, para contribuir con el aislamiento social por la covid 19 en el sector rural, además, solventar la necesidad de determinar si el nuevo modelo educativo está afectando el bienestar de los estudiantes y si esta afectación repercute en la disposición de los discentes hacia el estudio.
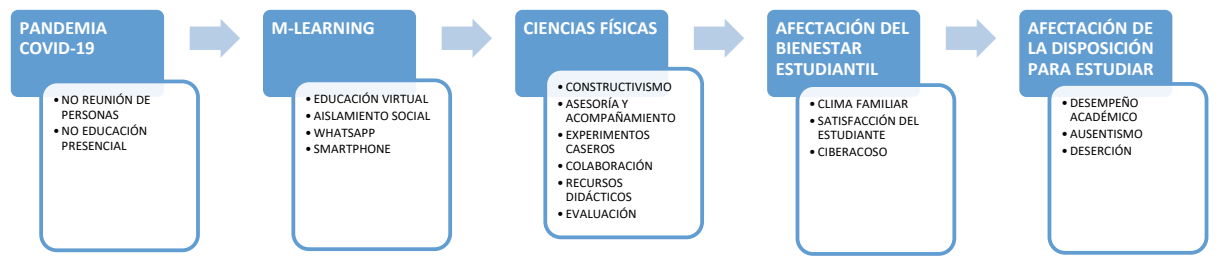

Figura 1. Planteamiento del problema

Fuente: elaboración propia.

Al poner en marcha el m-learning surgen las preguntas. ¿La implementación del modelo educativo m-learning en el colegio rural como contingencia por la pandemia de la covid-19 es efectiva para desarrollar el proceso de enseñanza-aprendizaje de la ciencia física? ¿Contribuye a reducir las interacciones físicas?, además, ¿Afecta el nuevo modelo educativo el bienestar de los estudiantes? ¿Repercute en la disposición de los discentes hacia el estudio?

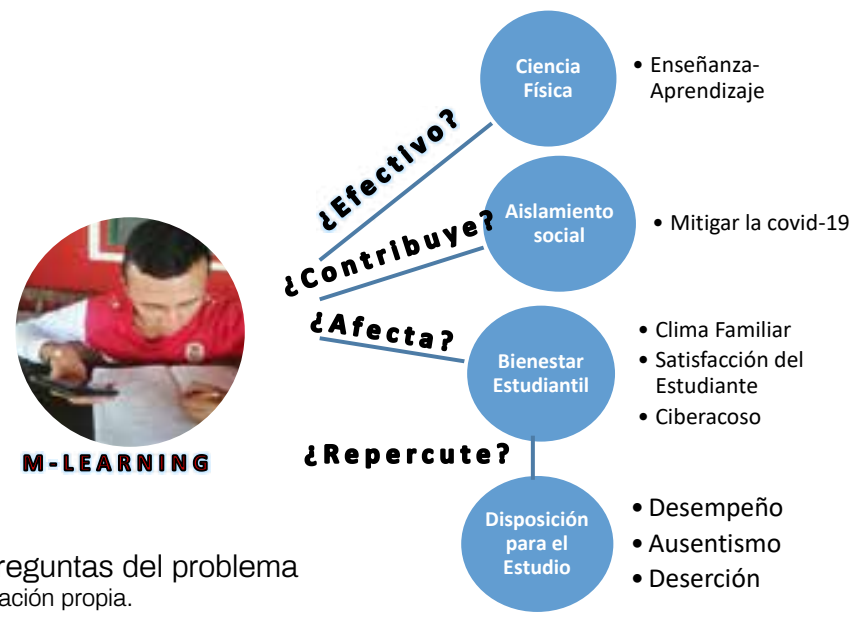

Figura 2. Preguntas del problema Fuente: elaboración propia. 
La primera faceta de la investigación presenta la aplicación de una estrategia educativa con el uso de las TIC para el sector rural, es así, que se implementa el modelo educativo m-learning como estrategia de contingencia por el covid-19. En ese sentido, el documento presenta las experiencias de la práctica docente con la estrategia educativa m-learning, que es la educación virtual con máxima portabilidad, interactividad y conectividad. Se trata de la integración del e-learning (sistema de enseñanza y aprendizaje a través de redes digitales) con los dispositivos móviles de comunicación, alcanzando experiencias educativas ubicuas.

El uso del teléfono móvil ofrece varias ventajas: un menor costo de adquisición, una menor exigencia de competencias digitales, como también la posibilidad de superar la barrera de la falta de acceso al servicio de energía eléctrica (Katz y Callorda, 2013). Por las características geográficas la portabilidad es fundamental en el contexto rural, la Organización de las Naciones Unidas para la Educación, la Ciencia y la Cultura (UNESCO, 2013) se refiere a su importancia cuando afirma que gracias a su facilidad para el transporte, los dispositivos móviles ofrecen la posibilidad de realizar diversas tareas, en particular aquellas relacionadas con la comunicación y el aprendizaje desde zonas apartadas.

WhatsApp es la aplicación de mensajería instantánea más popular del mundo. Usarla en educación, abre paso a novedosas metodologías como m-learning o b-learning y brinda una estrecha relación con lenguajes audiovisuales que la escuela hasta ahora no había logrado, permitiendo la creación de entornos virtuales de aprendizaje tanto online como offline, además, favorece la adquisición de las competencias tecnológicas (las llamadas e-competencias) fundamentales tanto en el terreno educativo como en el laboral. Reconocer las potencialidades educativas con la apropiación social de la web 2.0 desde la aplicación de mensajería instantánea WhatsApp, es también reconocer su aceptación y expansión.

Por sus ventajas el modelo m-learning ya se ha estado implementando en la región, Lugo et al. (2016) indican que al igual que en otras regiones del mundo, los países de América Latina han comenzado a implementar algunas alternativas del modelo de aprendizaje móvil centradas en la utilización de teléfonos inteligentes.

La segunda faceta de investigación, presenta un alcance descriptivo-causal. En esta faceta se desarrolla una investigación científica-social con enfoque educativo del impacto que ha tenido la implementación de la estrategia educativa m-learning durante la pandemia en la población estudiantil, que por su contexto es considerada vulnerable, y la repercusión de esa afectación en la educación. La necesidad se evidencia en el diagnóstico inicial donde se observa una indisposición hacia el estudio sin antecedentes para la región, se diligencia el diario de campo virtual con observaciones directas y una participación activa del autor por su condición de docente. Se quiere esclarecer la situación para luego poder brindar soluciones que mejoren los problemas existentes.

Esta propuesta supone considerar que en la educación no se debe tener en cuenta sólo lo académico. Es importante observar los marcos de referencia necesarios para posibilitar el desarrollo personal y social para así alcanzar las metas educativas. Se obtiene de la revisión de literatura, tres variables del bienestar estudiantil pertinentes, además, emerge una cuarta variable que es explicada por el bienestar estudiantil y está intrínsecamente relacionada con el aprendizaje. Para entender la 
dinámica de afectación se miden las correlaciones entre las variables del bienestar estudiantil: ambiente familiar, satisfacción de los estudiantes y victimización en la red, para luego, develar las posibles relaciones de causalidad de estas variables con la disposición hacia el estudio que es según lo que se observa, la variable donde desembocan los problemas del bienestar estudiantil. En otras palabras, la hipótesis de investigación expone: que si se está viendo afectada la disposición hacia el estudio, es el resultado de la afectación de las variables del bienestar estudiantil. El indicador de logro es entonces: la determinación de la existencia o no de las correlaciones entre las variables del bienestar estudiantil y las relaciones de causa entre estas y la disposición para el estudio.

El ambiente familiar en el m-learning. Los procesos para medir el ambiente escolar están naturalmente focalizados en el campus educativo, con el cambio de modelo se re-direcciona el enfoque hacia la familia. La afectación de uno de los renglones más sensibles del bienestar estudiantil como lo es el clima familiar es determinante en la disminución de la probabilidad de éxito escolar, más ahora que también corresponde al clima escolar explica la indisposición de los estudiantes. El clima familiar es abordado por Mendoza (2012) el cual asevera que la familia es un sistema complejo que contiene factores positivos y negativos que influyen al adolescente y pueden condicionar la deserción escolar. Sánchez (2015) corrobora la relación entre los diferentes aspectos de la familia con el rendimiento escolar. Correa (2018) comprueba la relación entre el funcionamiento familiar y la disposición para el estudio.

Es apropiado entonces para esta investigación medir el clima familiar en sus interrelaciones. El ambiente familiar y escolar es trascendental en el bienestar de los estudiantes, a lo que el Fondo de las Naciones Unidas para la Infancia (UNICEF, 2011) refiere que los adolescentes consideran importante desarrollar un sentido de aceptación y pertenencia de un grupo social.

El ambiente familiar está fuertemente ligado con los derechos fundamentales de los niños y adolescentes, en el sentido en que todo niño tiene derecho a un ambiente familiar seguro y pacífico, que en este tiempo de estudio desde casa promueva el aprendizaje y que todos los hijos puedan sentirse respetados, incluidos social y emocionalmente, además, se espera que tengan éxito escolar como parte de su proyecto de vida. La institución educativa desde el proyecto de bienestar institucional y toda la comunidad en general, deben trabajar juntos para fomentar y resguardar los derechos de los niños, niñas y adolescentes, apoyando a las familias en la búsqueda de su bienestar.

El cambio de modelo educativo afecta el clima familiar y su reacción no se hace esperar. En redes sociales las familias exteriorizan sus temores con la educación desde casa, memes por doquier donde se comenta con frases como "yo con quinto de primaria como le puedo ayudar a mi hijo que va en séptimo". Lo importante en el acompañamiento de las familias en el desarrollo de las actividades estudiantiles, es ayudar en la identificación de los errores y la supervisión. Puede ser que algunas veces las familias no puedan solucionar el error, pero les pueden ayudar a descubrir hasta donde han llegado y por qué se están equivocando para que con la revisión del grupo de WhatsApp, el repaso de los apuntes, la búsqueda de información y la orientación 
más personalizada del docente, se pueda superar ese error. La supervisión continua contribuye a forjar la autorregulación y que los discentes no sucumban ante la oferta distractora que presenta la internet.

En el proceso de aprendizaje, el identificar el error y superarlo es un avance significativo para alcanzar la competencia. De un fracaso se puede aprender, pero de un error se puede aprender mucho más, y es que por los intentos fallidos en los que se ha procedido de forma sólida, se han alcanzado muchos avances para la humanidad, además, nuestra capacidad de resiliencia se fortalece. "Profe yo no entiendo", esa pregunta no es consecuente a un estudiante que con su familia a tratado de desarrollar adecuadamente una actividad, la pregunta al docente debe ser puntual "profesor estoy cometiendo este error" y es ahí, donde se muestra una familia que verdaderamente ha acompañado al estudiante en su proceso de aprendizaje. Hay que disfrutar aprendiendo y esa felicidad debe brillar en el desarrollo de las actividades científicas desde casa en familia.

Ciberacoso en el m-learning. El empleo de WhatsApp no está exento de problemas éticos y de seguridad como el cyberbullying, grooming, sexting, phishing o los casos de suplantación de identidad, que afectan de manera sustancial el bienestar estudiantil. Esta afectación puede repercutir en indisposición hacia el estudio, se observa la pertinencia de la variable para esta investigación. Como una reacción a esta nueva realidad surge la infoética, que se emplea para referirse a los problemas éticos que está generando la sociedad de la información.

En un estudio sobre acoso escolar y clima familiar, Paredes (2016) estableció la existencia de una relación entre ambas variables. Su muestra estuvo conformada por adolescentes de edades entre 13 y 16 años en Perú.

El cyberbullying o ciberacoso escolar, es acoso escolar pero a través de internet y la UNICEF (2016) genera una alerta mundial al anunciar que ya se encuentra muy extendido, según su informe Poniendo fin al tormento: Abordando el acoso escolar desde el patio de la escuela hasta el ciberespacio; en el que participaron más de 100.000 niños y jóvenes de todo el mundo, se ilustra esta realidad con los siguientes datos:

Nueve de cada diez encuestados consideró que el ciberacoso escolar es un problema importante; dos tercios informaron haber sido víctimas; y un tercio creía que era normal y por lo tanto no se lo dijo a nadie, mientras que la mayoría no sabían a quién decirle o tenían miedo de hacerlo. Se detectó que el hostigar verbalmente es lo más frecuente y se evidencia, aún más, en las redes sociales y los grupos de WhatsApp. (Prefacio, p.v).

Calidad y satisfacción en la educación con el m-learning. La calidad del servicio educativo es subjetiva y representa satisfacción para el que lo recibe, por lo que en esta investigación para conocer la calidad del servicio educativo, se evalúa la satisfacción que representa este servicio para los estudiantes. Un estudiante aburrido es un estudiante insatisfecho con el servicio educativo, por esto, es importante reconocer que la satisfacción por el servicio educativo es un factor del ámbito escolar que influye en la percepción subjetiva de su propio bienestar. Al mismo tiempo, la satisfacción del estudiante influencia significativamente su disposición para estudiar. Se observa la pertinencia de la variable para este estudio. 
Lo competente que es un estudiante es un producto, se puede evaluar la calidad de ese producto de manera objetiva por ejemplo con la prueba SABER 11 para el caso de Colombia, pero la educación es un servicio no un producto. El servicio de educación es un concepto amplio que contiene intrínsecamente su efectividad y otros elementos que lo construyen para que su resultado sea integral. La educación entonces se debe abordar como un servicio, según Oliva y Pinzón (2012) la calidad de los productos puede ser más fácilmente medible que la de los servicios.

En los resultados obtenidos por Fernández (2017) indica que la calidad percibida influencia la satisfacción del estudiante y que las competencias del docente tienen repercusión en la satisfacción del discente en la escuela. El docente no se debe conformar con hacer lo mismo que ha hecho siempre, ahora desde una herramienta digital, sino que debe innovar, debe cautivar al estudiante para que el saber se adquiera con mayor naturalidad por este nativo digital. Al respecto Fonseca (2009) argumenta que: "Lo que vemos es que las prácticas presenciales se llevan a dichos ambientes sin mayor éxito, idéntica situación a la que vivieron los periódicos que migraron a la red y hacían visible la misma edición impresa sin mayores valores agregados" (p.4).

Por el estado de emergencia que se declaró a causa de la pandemia, los directivos de la educación envían instrucciones a las instituciones educativas de básica y media para incorporar una flexibilización en el proceso de evaluación, situación que conlleva a una respuesta atípica en los resultados académicos. Este hecho es interpretado por los estudiantes como un bajón de calidad, entienden que las instituciones no están en capacidad de hacer lo que es necesario, y por lo tanto, se recurre a la flexibilización.

Si el servicio educativo no satisface al estudiante desde su calidad, el efecto inmediato es que se aburre, luego se indispone, a mediano plazo deja de asistir a clases, para terminar como un desertor del sistema educativo. El servicio educativo no llena sus expectativas y deserta. Estos estudiantes insatisfechos, en el caso de que logren ser retenidos con chantajes o promesas, mantienen un alto ausentismo con una importante probabilidad de fracaso escolar. La situación también se puede mejorar con un seguimiento cercano interdisciplinario que trabaje en su proyecto de vida.

Disposición para el estudio en el m-learning. Cada vez cobra mayor importancia considerar el aprendizaje desde la perspectiva del estudiante, que es quien da significado y sentido a la información que procesa y es el que decide lo que quiere aprender, así como la manera de hacerlo. La variable disposición hacia el estudio involucra aspectos sencillos de índole afectivo y personal que intervienen en la regulación de la conducta dirigida hacia el estudio. Núñez (2009) señala que para aprender algo nuevo se requiere disposición. Como se ha dicho, la disposición hacia el estudio depende del bienestar estudiantil y al mismo tiempo, se relaciona intrínsecamente con el aprendizaje. Se observa el sentido y pertinencia para el análisis en la investigación.

Avena y Rivera (2013) encontraron una correlación significativa de 0,195 entre el funcionamiento familiar con una de las dimensiones de la disposición para el estudio como es la motivación escolar en estudiantes de secundaria mexicanos. Fernández (2017) encontró que la satisfacción del estudiante influencia significativamente su motivación y que la motivación del estudiante influencia las intenciones de graduación disminuyendo la deserción. 
Según Panimboza (2017) el bullying o acoso escolar es un factor para la apatía escolar, disminuir y controlar el ciberacoso logra impulsar el aprendizaje en los adolescentes y mejorar su rendimiento.

La apatía puede impedir que se desarrollen las habilidades cognitivas de los estudiantes, más aun en el modelo de aprendizaje m-learning donde el autoaprendizaje es un factor fundamental, se requiere de una autorregulación académica. Según Baumert et al. (1999) el aprendizaje autorregulado se entiende como la capacidad de desarrollar conocimiento, habilidades y actitudes que facilitan el conocimiento a futuro. Es indispensable entonces, desarrollar un metaaprendizaje de antemano. Birenbaum (2002) expresa que el aprendizaje autodirigido implica la capacidad de asimilar nuevo conocimiento y aplicarlo en la solución de problemas, la habilidad para pensar críticamente y poner en funcionamiento la autoevaluación, así como comunicarse y colaborar con otros. Como se ha observado, en el aprendizaje autónomo los alumnos son metacognitivamente, motivacionalmente y conductualmente activos en su aprendizaje. Chiappe y Romero (2018) comprobaron la falta de autodisciplina para usar los dispositivos de comunicación en actividades académicas.

\section{Métodos y materiales}

Diseño metodológico. En la primera faceta la investigación es aplicada, se presentan reflexiones y experiencias de la práctica docente en el m-learning con énfasis en la ciencia física. Para la segunda faceta la investigación es científica-social con enfoque educativo, cualitativa y no experimental. El diseño es transeccional. La hipótesis es descriptivo-causal. Se tiene la posibilidad de definir las variables independientes y la dependiente. La hipótesis es estadística, es decir, concreta y operativa. La muestra es representativa de la población. El proceso de recolección, procesamiento y análisis de datos se desarrolla desde el paradigma cuantitativo. Los instrumentos para la recolección de datos son: el diario de campo virtual y la encuesta virtual. Se hace uso de cuatro pruebas validadas para el tipo de población. El análisis de resultados se direcciona con prioridad de peso causal prospectivo. (Hernández et al., 2010).

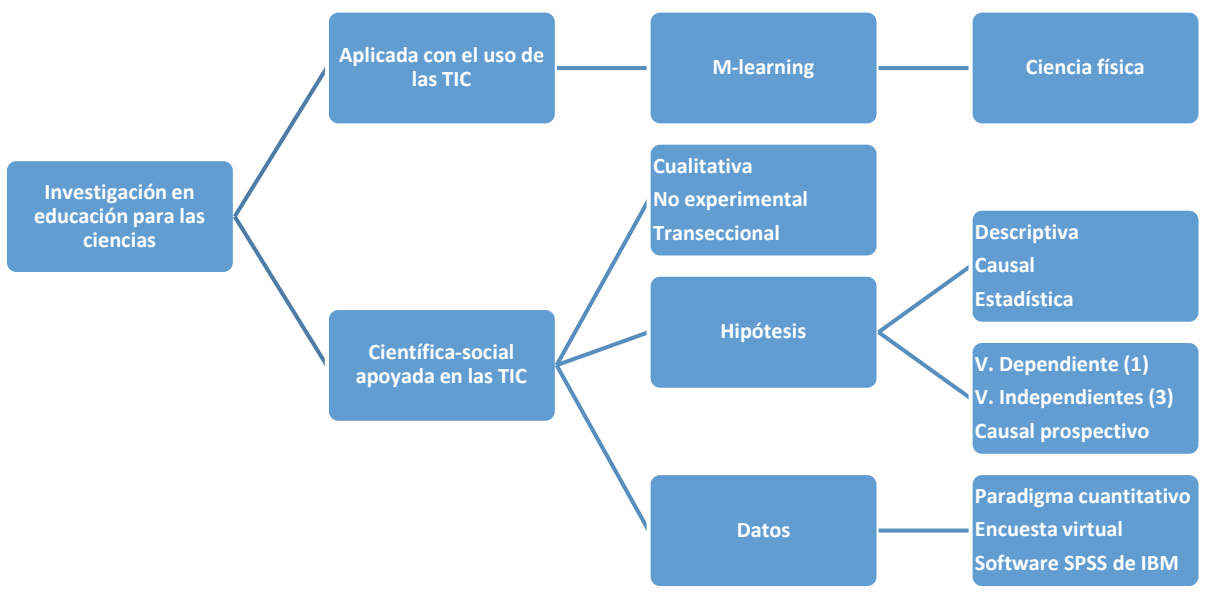

Figura 3. Diseño Metodológico

Fuente: elaboración propia. 
Instrumentos de medida. La Escala de Clima Familiar (FES). La escala dicotómica fue creada por Moos y Moos (1981). Es una escala estandarizada para adolescentes de secundaria por Ruiz y Guerra (1993) en Lima, Perú. La dimensión Relaciones que es la que se usa para esta investigación, evalúa el grado de comunicación y libre expresión dentro de la familia así como el grado de interacción conflictiva, tiene 27 reactivos en total con tres sub-escalas (cohesión, expresividad y conflicto).

La Escala de Victimización entre Adolescentes a través del Teléfono Móvil y de Internet (CYB-VIC). Utiliza una escala tipo Likert desarrollada por Buelga et al. (2012), se accede a la versión abreviada de 7 reactivos validada por Gálvez-Nieto et al. (2016) en Chile. La escala mide el acoso experimentado a través del teléfono móvil y de internet durante el último año. Los cálculos se desarrollan con los datos transformados, hecho que es significativo para el sentido de las respuestas, se debe hablar ahora de una escala que mide no la percepción de victimización por el cyberbullying sino la sensación de seguridad en la red.

La Escala de Satisfacción del Discente (ISC). Se empleó el cuestionario de Satisfacción Intrínseca con la escuela, del Intrinsic Satisfaction Classroom Scale (ISC) Nicholls et al. (1985). Este instrumento tipo Likert, presenta ocho ítems que miden el grado de satisfacción con la escuela, con dos subescalas, que miden la satisfacción/ diversión y el aburrimiento.

La Escala de Disposición hacia el Estudio. El constructo fue propuesto por Escobedo et al. (2011) para estudiantes del sur de México. Mide y diagnóstica la disposición hacia el estudio de alumnos de educación secundaria. El constructo contiene 19 preguntas, con escala tipo Likert. Las dimensiones que se desarrollan son: motivación intrínseca hacia el estudio, compromiso con la tarea, autorregulación y adaptación.

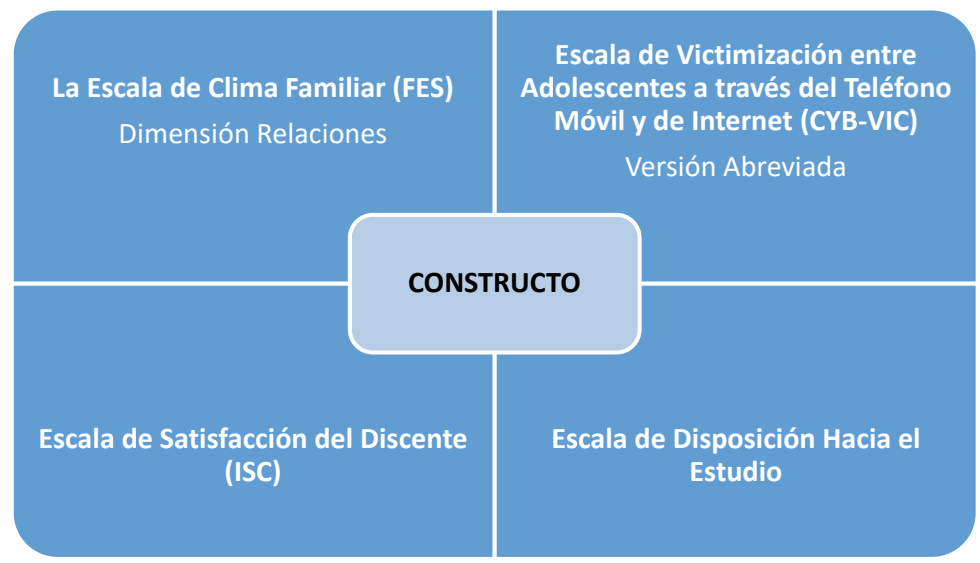

Figura 4. Instrumento para la recolección de datos Fuente: Elaboración propia.

Población y muestra. La población objeto de estudio son los 464 estudiantes de secundaria del colegio público rural. El marco muestral cuenta con representación de todos los grupos de la población y su tamaño es de 450 estudiantes. Para determinar el tamaño de la muestra, en esta investigación se establece un nivel de confianza del 
$95 \%$, con un margen de error del $8 \%$ bajo el supuesto de máxima indeterminación $p=q=50 \%$. Según los cálculos, se decide seleccionar una unidad de análisis de 116 casos. La muestra corresponde al $25 \%$ de la población y al 25,8\% del marco muestral. Por la pandemia la selección de la muestra se desarrolla a conveniencia y guiada, asegurando la representación de todos los grupos poblacionales. La encuesta se desarrolla con el software de formularios de google.

\section{Resultados}

Faceta 1. La experiencia significativa de la práctica docente desde el constructivismo acude a desarrollos con los que los alumnos se comprometen activamente mediante interacción social. Esto posibilita que el estudiante pase de ser una persona pasiva, a un sujeto activo dentro del proceso de enseñanza-aprendizaje, apoyado por un docente que cumple el rol de guía. Claramente el uso de las TIC influye de manera significativa en la pedagogía conduciéndola inexorablemente a una especialización del paradigma hacia el conectivismo, modificando no solo la forma de aplicar sus secuencias didácticas sino también en sus contenidos. Desde el m-learning, los teléfonos móviles deben permitir hacer uso de lo que el estudiante sabe y conoce para que la búsqueda o recepción de información permita construir, sustentar o modificar el conocimiento.

El conectivismo se relaciona estrechamente con el constructivismo al señalar que el aprendizaje es un proceso de formación colaborativa y social. La relación del $m$-learning con el conectivismo es intrínseca por las asociaciones con los dispositivos móviles. Es importante el empleo de métodos y estrategias que permitan a los alumnos desde el teléfono móvil explorar temas y entornos complejos activamente, el estudio de los contenidos en su contexto, la construcción del conocimiento y su posterior discusión con otras personas para validarlos.

Se está desarrollando el m-learning, las clases de ciencias se llevan a cabo con el teléfono móvil a través de la aplicación de mensajería instantánea WhatsApp, los estudiantes aprovechan la ubicuidad para recibir las orientaciones educativas en sus hogares. Se establecen grupos de WhatsApp académicos y para las actividades de dirección de grado. Los experimentos de ciencia física se desarrollan desde casa. Para el curso se diseña un programa educativo en m-learning y una guía de orientación.

Tabla 1. Diseño de un programa educativo en m-learning (muestra)

Sección general del curso en m-learning

\begin{tabular}{|c|c|}
\hline \multicolumn{2}{|c|}{ Identificación del curso } \\
\hline Datos del docente & $\begin{array}{l}\text { Docente } \\
\text { WhatsApp \# } \\
\text { e-mail }\end{array}$ \\
\hline Nombre del curso & $\begin{array}{l}\text { CIENCIA FÍSICA } \\
\text { LA LUZ }\end{array}$ \\
\hline $\begin{array}{l}\text { Año lectivo - Gra- } \\
\text { do }\end{array}$ & 2020 - Grado 11 - Periodo 2 \\
\hline $\begin{array}{l}\text { Número de se- } \\
\text { manas }\end{array}$ & 9 semanas \\
\hline $\begin{array}{l}\text { Intensidad horaria } \\
\text { semanal }\end{array}$ & 3 horas \\
\hline
\end{tabular}




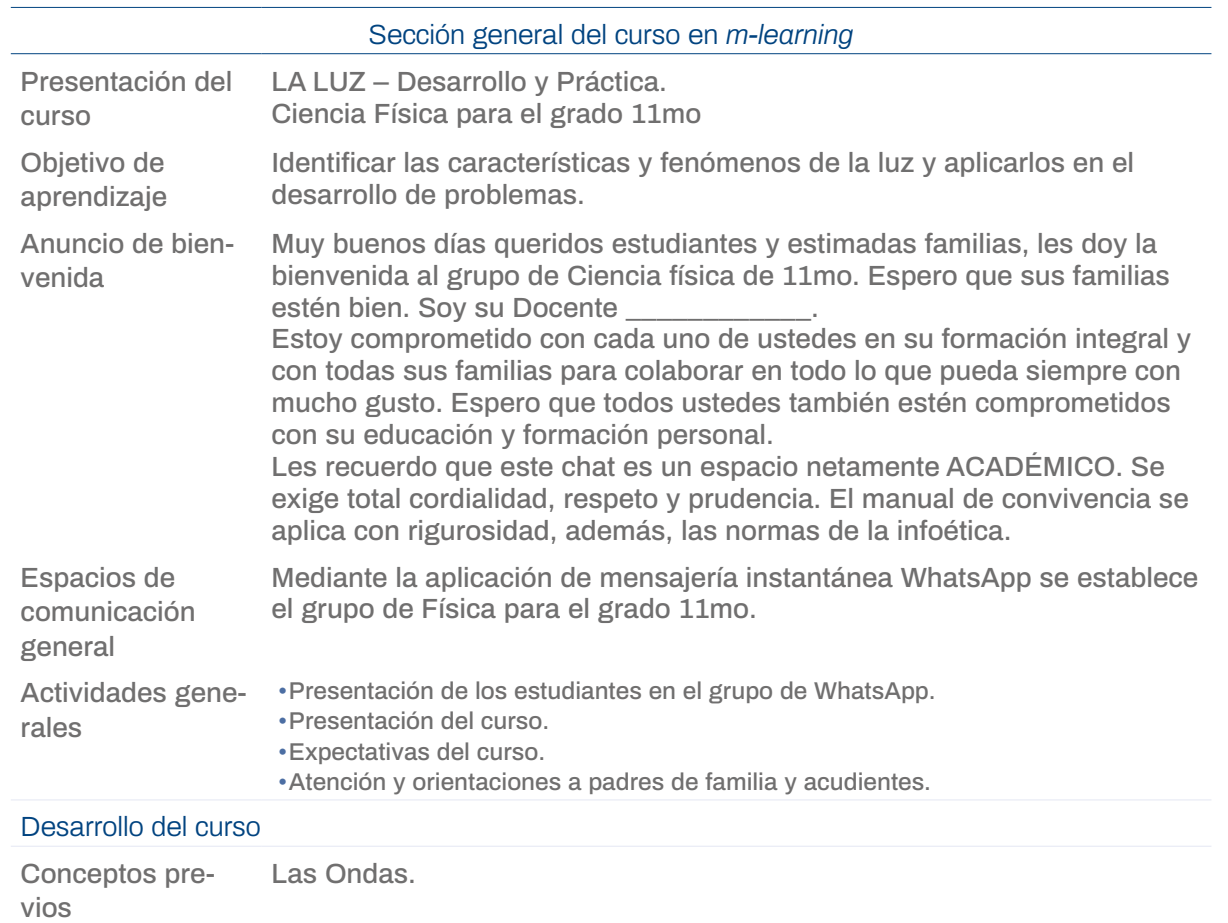

Competencia a Identifica las características y fenómenos de la luz con base en el estudio desarrollar del fenómeno ondulatorio y los aplica en el desarrollo de problemas.

Contenidos temá- Activación de presaberes.

ticos $\quad$ Propagación de la luz. Experimento 1. Taller de aplicación 1.

- Velocidad de la luz. Experimento 2. Taller de aplicación 2.

- Las sombras. Experimento 3. Taller de aplicación 3.

-Difracción de la luz. Experimento 4. Taller de aplicación 4.

-Espectro visible. Experimento 5. Taller de aplicación 5.

-Reflexión de la luz. Experimento 6. Taller de aplicación 6.

-Refracción de la luz. Experimento 7. Taller de aplicación 7.

Recursos didác- Imágenes, Videos, Textos, Audios y Actividades interactivas online.

ticos

Actividades de https://es.khanacademy.org/join/4VA5YEMD

Retroalimentación Con el enlace podrán registrarse en Khan Academy y formar parte de los estudiantes de la clase, donde encontrarán las actividades de refuerzo y retroalimentación.

Evaluación del curso

Evaluación Formativa - Permanente.

Tipo de evidencia Conocimiento, desempeño y producto.

Descripción La guía se desarrolla sobre el imprimible. A los desarrollos se les toma fotografía y se envía al contacto privado del WhatsApp del docente. El estudiante sustenta el desarrollo de la guía donde se harán preguntas durante: la explicación del docente, el desarrollo de los experimentos y durante los talleres en el grupo de WhatsApp, además, se toma asistencia virtual.

Evidencia entre- Fotografía de las actividades desarrolladas y respuestas que sustenten gable su desarrollo. 


\section{Sección general del curso en m-learning}

Criterios de evaluación

Autoevaluación

Criterios de autoevaluación

Superior. Si el estudiante ha cumplido con todos los parámetros académicos de entrega, con buena calidad en todos sus desarrollos (destacado), en el tiempo establecido y su comportamiento ha sido excelente.

Alto. Si el estudiante ha cumplido con todos los parámetros académicos de entrega, con aceptable calidad, en el tiempo establecido y su comportamiento ha sido excelente.

Bajo. Si el estudiante no ha cumplido con ningún parámetro académmico de entrega.

Nota: el aporte colaborativo de WhtsApp que sea registrado por el docente, mejorará el nivel de desempeño del estudiante.

El curso en m-learning concibe este proceso como un ejercicio permanente de autorreflexión, autoanálisis y autocrítica constructiva, encaminado esencialmente a generar una cultura de calidad y autorregulación que garantice el mejoramiento continuo.

Se plantea con este objetivo la siguiente actividad de ayuda para la reflexión propia del proceso de autoevaluación.

Superior. Si el estudiante encuentra que: ha cumplido con todos los parámetros de académicos de entrega, con disciplina, honestidad y responsabilidad. Que ha fomentado un buen ambiente escolar, tanto en la familia como en el grupo WhatsApp, integrándose, participando en clase y mostrando interés por fomentar un aprendizaje colaborativo.

Alto. Si el estudiante encuentra que: Hacumplido con algunos parámetros académicos de entrega con honestidad. Que ha mantenido un buen ambiente escolar, tanto en la familia como en el grupo WhatsApp.

Bajo. Si el estudiante encuentra que: No hacumplido con ningún parámetro académico o de ambiente escolar.

\begin{tabular}{|l|l|l|l|l|}
\hline & Siempre & $\begin{array}{c}\text { La mayoría } \\
\text { de las veces }\end{array}$ & $\begin{array}{c}\text { Ocasional- } \\
\text { mente }\end{array}$ & Pocas veces \\
\hline $\begin{array}{l}\text { Fomenté un buen ambiente en mi } \\
\text { familia y en el grupo }\end{array}$ & & & & \\
\hline Cumplí con los plazos de entrega & & & & \\
\hline $\begin{array}{l}\text { Aportéal grupo de WhatsApp ideas, } \\
\text { fuentes de consulta, otros. }\end{array}$ & & & & \\
\hline Cuidé los materiales de trabajo & & & & \\
\hline $\begin{array}{l}\text { Expuse mis ideas, preguntasy pun- } \\
\text { tos de vista de manera respetuosa } \\
\text { y prudente }\end{array}$ & & & & \\
\hline $\begin{array}{l}\text { Contribuí a que otros también } \\
\text { aprendieran }\end{array}$ & & & & \\
\hline $\begin{array}{l}\text { Escuché y valoré el trabajo de mis } \\
\text { compañeros }\end{array}$ & & & & \\
\hline Llevé todos los desarrollos "al día" & & & & \\
\hline $\begin{array}{l}\text { ¿En qué aspecto del desarrollo de } \\
\text { las actividades me destaco? }\end{array}$ & & & & \\
\hline Qué debo mejorar & & & & \\
\hline
\end{tabular}

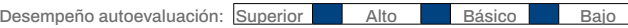

Fuente: Elaboración propia.

Entre las actividades didácticas que se llevan a cabo con la aplicación de mensajería instantánea WhatsApp están: explicaciones con texto, audio, imagen y audiovisuales, donde los docentes imparten clases con secuencias didácticas definidas y los estudiantes pueden interactuar de manera colaborativa, fomentando el dialogo constructivo y la comunicación docente-estudiante-familia. 


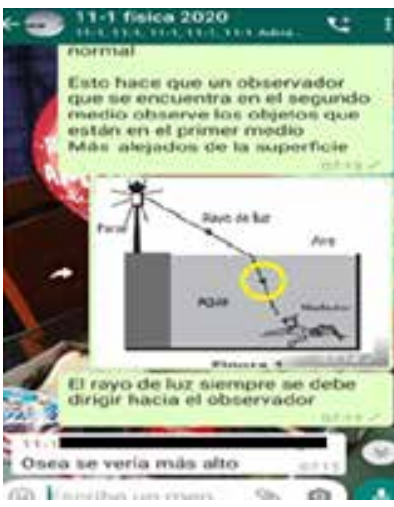

Figura 5. Orientaciones de Ciencia Física en el m-learning Fuente: Elaboración propia.

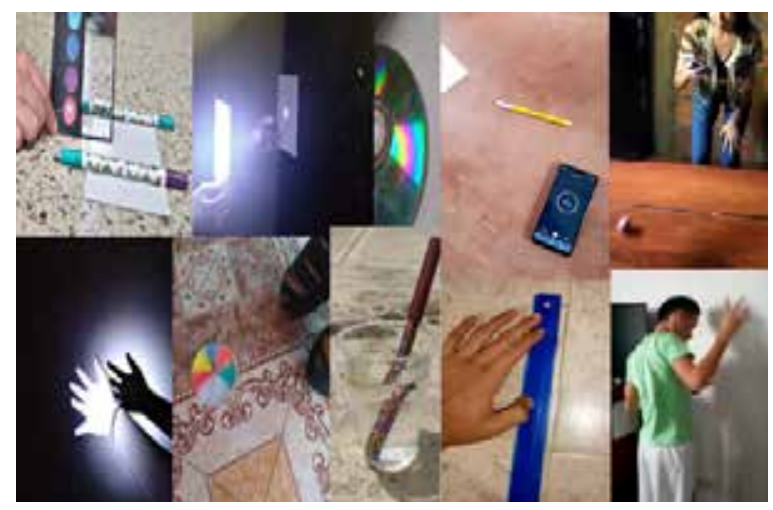

Figura 6. Experimentos caseros de Ciencia Física en el m-learning

Los experimentos caseros son una estrategia didáctica que desde el paradigma constructivista promueve el desarrollo de competencias científicas. Los estudiantes necesitan experimentar para aprender cosas nuevas o reforzar las ya aprendidas, el no poder asistir al laboratorio escolar dificulta su aprendizaje. La interacción y la socialización con los docentes y compañeros de clase cambió, ahora se realiza mediante la aplicación de mensajería instantánea WhatsApp, esto deriva en distractores que ofrece la internet en una clase en línea. Sin embargo, el m-learning permite que los estudiantes puedan tener una experiencia vivencial con materiales que tienen en casa, variando el estímulo a una práctica física que capta mejor la atención, a la vez que los estudiantes aprenden de forma experiencial y divertida, se evidencia que es un ambiente inmersivo con contenido cautivador hacia la ciencia. Preparar los materiales, realizar el experimento o tomar fotografías a lo largo del proceso es constructivo. Además, en algunos casos los estudiantes repitieron el experimento varias veces para observar lo que sucedía o mejorar sus resultados. En un mensaje después de la clase de práctica casera, un estudiante expresó: "Los experimentos estuvieron divertidos y en casa todos me ayudaron, me siento feliz".

Según Mancinas (2018) el m-learning privilegia una cognición de tipo empírico, centrada en la ejecución de procedimientos que oscilan entre una actividad mental de tipo algorítmico y otra de tipo heurístico.

Los resultados de la evaluación fueron reveladores, se logró fortalecer en la mayoría de los educandos las destrezas y la comprensión de los conceptos de la ciencia física. Las prácticas caseras, concebidas como una estrategia didáctica para la enseñanza y aprendizaje de la ciencia física, permiten el desarrollo de habilidades científicas y un aprendizaje más significativo.

En los contextos académicos rurales se observa que el Smartphone y el aplicativo WhatsApp se destacan por la movilidad y las ventajas económicas de acceso. Se comprueba que el m-learning es efectivo para el proceso de enseñanza-aprendizaje de la ciencia física. En este sentido, Zapata (s.f.) al realizar una sesión de aprendizaje en m-learning con WhatsApp, concluye que permite la construcción del 
conocimiento mejorando la comunicación, alcanzándose el logro previsto.

Faceta 2. En el diagnóstico inicial, al diligenciar el diario de observaciones de campo virtual se muestra que la implementación del m-learning en la pandemia ha develado la problemática social intrincada en estas zonas apartadas, los estudiantes reaccionan mostrando una alta indisposición hacia el estudio. Las apreciaciones expuestas por el cuerpo docente, estudiantes y familiares han evidenciado que un importante grupo de estudiantes no tiene disposición para estudiar, están apáticos. El alto nivel de indisposición es inédito para el colegio, siendo este, el factor referencial para esta segunda faceta del artículo. Las expectativas educativas de esta generación están en riesgo y la brecha con el sector urbano se podría incrementar.

Los hechos descritos en el diario de campo virtual, corresponden a manifestaciones por llamada telefónica, por la aplicación de mensajería instantánea WhatsApp o reunión de docentes en Zoom.

Tabla 2. Diario de observaciones de campo virtual (muestra)

\begin{tabular}{|c|c|c|c|}
\hline Fecha & Interviniente & Situación & Descripción \\
\hline $\begin{array}{l}\text { Abril } \\
2020\end{array}$ & Acudiente & $\begin{array}{l}\text { Dirección de } \\
\text { grado }\end{array}$ & $\begin{array}{l}\text { Mi hijo me dice que se está conectandoa las clases } \\
\text { y está enviando todo, veo que es mentira. Yo le } \\
\text { recargo el celular y me voy a trabajar, no puedo } \\
\text { supervisarlo. Voy a revisarle el WhatsApp y ver } \\
\text { por qué no se conecta. }\end{array}$ \\
\hline $\begin{array}{l}\text { Abril } \\
2020\end{array}$ & Docente & Reunión & $\begin{array}{l}\text { Los estudiantes no quieren hacer nada. } \\
\text { No envían las evidencias. }\end{array}$ \\
\hline $\begin{array}{l}\text { Mayo } \\
2020\end{array}$ & Docente & Reunión & $\begin{array}{l}\text { Los padres no les colaboran con las acti- } \\
\text { vidades a los estudiantes. }\end{array}$ \\
\hline $\begin{array}{l}\text { Mayo } \\
2020\end{array}$ & Estudiante & $\begin{array}{l}\text { Seguimien- } \\
\text { to }\end{array}$ & $\begin{array}{l}\text { Me toca ir a trabajar, si no trabajo no como, } \\
\text { toca colaborar en la casa también, no tengo } \\
\text { ganas de estudiar. }\end{array}$ \\
\hline $\begin{array}{l}\text { Junio } \\
2020\end{array}$ & Docente & Consulta & $\begin{array}{l}\text { Los estudiantes sólo se quejan y sacan } \\
\text { excusas pero no quieren estudiar. }\end{array}$ \\
\hline $\begin{array}{l}\text { Junio } \\
2020\end{array}$ & Estudiante & $\begin{array}{l}\text { Seguimien- } \\
\text { to }\end{array}$ & $\begin{array}{l}\text { En mi casa pelean mucho, no entiendo } \\
\text { algunas clases, no me gusta estudiar así. }\end{array}$ \\
\hline $\begin{array}{l}\text { Julio } \\
2020\end{array}$ & Docente & Reunión & $\begin{array}{l}\text { Los estudiantes casi no se están conectando a } \\
\text { las clases, sólo envían al final del período y con } \\
\text { baja calidad. }\end{array}$ \\
\hline $\begin{array}{l}\text { Julio } \\
2020\end{array}$ & Estudiante & $\begin{array}{l}\text { Dirección } \\
\text { de grado }\end{array}$ & $\begin{array}{l}\text { Leer sólo en casa, es aburrido. Quiero } \\
\text { estar en el colegio. }\end{array}$ \\
\hline
\end{tabular}

Fuente: elaboración propia.

Los procedimientos para la obtención de los resultados se desarrollan con el apoyo del software SPSS Statistics de IBM. 
Análisis de las características psicométricas

Confiabilidad. Método de Medidas de Coherencia o Consistencia Interna. Alfa de Cronbach (escalas tipo Likert). Coeficientes KR-20 y KR-21 (escala Dicotómica). Se establece con la prueba de contraste. Para las cuatro escalas y el instrumento completo se acepta la Ha. El coeficiente a es de aceptable a bueno, las escalas y el instrumento completo presentan una buena consistencia interna. La supresión de ningún reactivo aumenta significativamente el coeficiente $\alpha$.

Validez. Método Análisis Factorial de Varianza Total. Técnica Análisis de Componentes Principales (ACP). La interpretación se hace a partir de la matriz de saturaciones que resulta de la rotación de los ejes por el método Varimax.

Evaluación del ACP. Para las cuatro escalas se encuentra que los resultados evidencian que el método ACP es adecuado para validar su constructo.

Interpretación del ACP. Se establece según la evidencia, que las cuatro escalas presentan validez de constructo para el contexto de esta investigación.

Prueba de Normalidad. La prueba Kolmogorov - Smirnov es adecuada para el tamaño la muestra. La distribución de la muestra es no normal $p<0,05$; se justifica el empleo de la estadística no paramétrica en la contrastación de hipótesis.

\section{Estadígrafos}

Análisis de Correlaciones. Se mide con los coeficientes de Kendall y de Spearman. Se muestran los resultados con el coeficiente Rho de Spearman.

Análisis de Causalidad. La variable explicada corresponde a la disposición hacia el estudio. La relación de causa se determina con el coeficiente D de Sommers. Correlaciones

Tabla 3. Coeficiente de correlación Rho de Spearman

\begin{tabular}{|c|c|c|c|c|c|}
\hline \multicolumn{6}{|c|}{ Correlaciones } \\
\hline & & & FES & CYB-VIC & ISC \\
\hline \multirow{7}{*}{$\begin{array}{l}\text { Rho de Spear- } \\
\text { man }\end{array}$} & \multirow[t]{2}{*}{ FES } & Coeficiente de correlación & 1,000 &, $239 * *$ &, $294^{* *}$ \\
\hline & & Sig. (bilateral) & . & ,010 &, 001 \\
\hline & \multirow[t]{2}{*}{ CYB-VIC } & Coeficiente de correlación & ,239** & 1,000 &, 129 \\
\hline & & Sig. (bilateral) & 010 & . & , 168 \\
\hline & \multirow[t]{3}{*}{ ISC } & Coeficiente de correlación & ,294** & ,129 & 1,000 \\
\hline & & Sig. (bilateral) & ,001 & ,168 & . \\
\hline & & $\mathrm{N}$ & 116 & 116 & 116 \\
\hline \multicolumn{6}{|c|}{ *. La correlación es significativa en el nivel 0,05 (bilateral). } \\
\hline \multicolumn{6}{|c|}{ **. La correlación es significativa en el nivel 0,01 (bilateral). } \\
\hline
\end{tabular}

Resultados de la correlación FES con CYB-VIC. Se acepta la Ha. Existe asociación muy significativa y positiva entre las dos variables del 23,9\%.

Resultados de la correlación FES con ISC. Se acepta la Ha. Existe asociación muy significativa y positiva entre las dos variables del $29,4 \%$. 
Resultados de la correlación CYB-VIC con ISC. Se acepta la Ho. No existe asociación significativa entre las variables.

\section{Causalidad}

Tabla 4. Coeficiente d de Sommers entre FES y disposición hacia el estudio

\begin{tabular}{|c|c|c|c|c|}
\hline \multicolumn{5}{|c|}{ Medidas direccionales } \\
\hline & & $\begin{array}{l}\text { Error estándar } \\
\text { asintótico }^{a}\end{array}$ & T aproximada ${ }^{b}$ & $\begin{array}{l}\text { Significación } \\
\text { aproximada }\end{array}$ \\
\hline \multirow{3}{*}{$\begin{array}{ll}\text { Ordinal por } & \mathrm{d} \text { de Som- } \\
\text { ordinal } & \text { mers }\end{array}$} & Simétrico & ,075 & 3,134 & ,002 \\
\hline & FES dependiente & ,074 & 3,134 & ,002 \\
\hline & $\begin{array}{l}\text { Disposición depen- } \\
\text { diente }\end{array}$ & ,075 & 3,134 & ,002 \\
\hline \multicolumn{5}{|c|}{ a. No se presupone la hipótesis nula. } \\
\hline \multicolumn{5}{|c|}{ b. Utilización del error estándar asintótico que presupone la hipótesis nula. } \\
\hline
\end{tabular}

Fuente: Elaboración propia.

Tabla 5. Coeficiente d de Sommers entre ISC y disposición hacia el estudio

\begin{tabular}{|c|c|c|c|c|}
\hline \multicolumn{5}{|c|}{ Medidas direccionales } \\
\hline & & $\begin{array}{c}\text { Error } \\
\text { estándar } \\
\text { asintótico }^{a}\end{array}$ & T aproximada ${ }^{b}$ & $\begin{array}{l}\text { Significación } \\
\text { aproximada }\end{array}$ \\
\hline \multirow{3}{*}{$\begin{array}{ll}\text { Ordinal por } & \mathrm{d} d \mathrm{de} \\
\text { ordinal } & \text { Sommers }\end{array}$} & Simétrico & ,059 & 8,491 &, 000 \\
\hline & ISC dependiente & ,061 & 8,491 & ,000 \\
\hline & $\begin{array}{l}\text { Disposición depen- } \\
\text { diente }\end{array}$ &, 057 & 8,491 &, 000 \\
\hline \multicolumn{5}{|c|}{ a. No se presupone la hipótesis nula. } \\
\hline \multicolumn{5}{|c|}{ b. Utilización del error estándar asintótico que presupone la hipótesis nula. } \\
\hline
\end{tabular}

Tabla 6. Coeficiente d de Sommers entre CYB-VIC y disposición hacia el estudio

\begin{tabular}{|c|c|c|c|c|c|}
\hline \multicolumn{6}{|c|}{ Medidas direccionales } \\
\hline & & & $\begin{array}{c}\text { Error } \\
\text { estándar } \\
\text { asintótico }\end{array}$ & $\begin{array}{c}\mathrm{T} \\
\text { aproximada }^{\mathrm{b}}\end{array}$ & $\begin{array}{l}\text { Significación } \\
\text { aproximada }\end{array}$ \\
\hline \multirow{3}{*}{$\begin{array}{l}\text { Ordinal por } \\
\text { ordinal }\end{array}$} & \multirow{3}{*}{$\begin{array}{l}\text { d de } \\
\text { Som- } \\
\text { mers }\end{array}$} & Simétrico & 072 &,- 178 & ,858 \\
\hline & & CYB-VIC dependiente & ,049 &,- 178 & ,858 \\
\hline & & $\begin{array}{l}\text { Disposición depen- } \\
\text { diente }\end{array}$ &, 139 &,- 178 & ,858 \\
\hline \multicolumn{6}{|c|}{ a. No se presupone la hipótesis nula. } \\
\hline \multicolumn{6}{|c|}{ b. Utilización del error estándar asintótico que presupone la hipótesis nula. } \\
\hline
\end{tabular}

Fuente: Elaboración propia.

Entre la variable explicada Disposición hacia el estudio con la variable independiente FES. Se acepta la Ha. Existe relación causal significativa $(p<0,05)$, existe relación simétrica y la relación asimétrica es positiva del 24,1\%. 
Entre la variable explicada Disposición hacia el estudio con la variable independiente ISC. Se acepta la Ha. Existe relación causal muy significativa $(p<0,01)$, existe relación simétrica y la relación asimétrica es positiva del 51\%.

Entre la variable explicada Disposición hacia el estudio y la independiente CYBVIC. Se acepta la Ho. No existe relación causal significativa $(p>0,05)$.

\section{Discusión}

La estrategia pedagógica m-learning se está aplicando en el colegio rural, el nuevo modelo educativo cumple con el objetivo de desarrollar el proceso de enseñanzaaprendizaje de la ciencia física con efectividad en el lugar donde se encuentran los discentes contribuyendo con el aislamiento social para contener la covid-19. Ruiz y Martínez (2012) afirman que los contextos de aprendizaje se van modificando en torno a las demandas sociales y a los múltiples medios tecnológicos que transcienden en la vida cotidiana.

El m-learning desde la corriente pedagógica del constructivismo conectivista, señala que el aprendizaje móvil es un proceso de formación colaborativo y social. En la relación intrínseca del m-learning con el conectivismo en la asociación con los dispositivos móviles, con el constructivismo el aprendizaje será una actividad que el sujeto realiza desde su experiencia con el entorno, esta vez apoyado del teléfono móvil, donde el desarrollo cognoscitivo y las funciones elementales que intervienen como la dialéctica, son una constante en el proceso de enseñanza-aprendizaje. Las prácticas caseras, concebidas como una estrategia didáctica para la enseñanza-aprendizaje de la ciencia física, permiten el desarrollo de competencias científicas y un aprendizaje más significativo. Ruiz y Martínez (2012) indican que el aprendizaje se alcanza a través de los procesos de intercambio e interacción social, que permiten construir y reconstruir conocimientos a través de estrategias colaborativas donde en escenarios virtuales surten un mayor sentido, ya que los procesos de comunicación y discusión requieren de un modelo que favorezca la interactividad colectiva.

La disposición hacia el m-learning es determinante para que la estrategia de enseñanza-aprendizaje m-learning sea exitosa desde los resultados académicos. Un estudiante apático presenta alta probabilidad de fracaso escolar. Se espera por la evidencia del diagnóstico inicial, un aumento en la reprobación, es indispensable institucionalizar la flexibilización académica y fomentar el aprendizaje autónomo. El docente debe cautivar a los estudiantes desde la didáctica, este debe innovar para que el alfabeta digital que es el estudiante, aumente su disposición hacia el estudio. Beltrán (1993) encuentra que partiendo de la evidencia de que el aprendizaje es un proceso socialmente mediado, también es necesario precisar que requiere una implicación activa del estudiante, única manera de que se produzca un cambio real en la comprensión significativa.

La dimensión Relaciones en la familia y la dimensión Satisfacción del discente se influyen bilateralmente en un $30 \%$. De afectarse el clima familiar en sus interrelaciones, se afecta también el ambiente escolar, ya que la educación se da desde casa y en consecuencia se afecta la percepción de calidad que tiene el discente por el servicio educativo y viceversa. Es importante para el m-learning reconocer esta simbiosis donde las soluciones se deben dar para las dos dimensiones simul- 
táneamente. La dimensión Relaciones en la familia está asociada con la dimensión percepción de seguridad en la red y el teléfono móvil. La dimensión Relaciones en la familia y la dimensión percepción de seguridad en internet se influyen bilateralmente en un $24 \%$. Si se afecta la sensación de seguridad por ciberacoso, también se afecta el clima familiar en sus interrelaciones y viceversa. La dimensión Ciberacoso no está asociada con la dimensión Satisfacción del discente. Ha sido mitigado el fenómeno del ciberacoso entre estudiantes, razón por la que los discentes no la vinculan a la educación. Paredes (2016) encuentra una relación significativa entre el acoso escolar y el clima familiar de $-0,124$.

La variable ciberacoso no explica el comportamiento de la variable disposición hacia el estudio, hay independencia entre las variables. El peligro de ciberacoso ha sido controlado y mitigado, los estudiantes presentan sensaciones de seguridad en la red. La indisposición hacia el estudio no es consecuencia del ciberacoso. Además, como se observa en los resultados de correlación, los estudiantes no relacionan el riesgo de seguridad en la red con la educación. Ortega y González (2016) sugieren que existe una relación entre el ciberacoso y el rendimiento académico.

Dentro de las consecuencias que trae un inadecuado clima familiar se tiene la indisposición para desarrollar las actividades académicas. Este hecho se puede observar en que la dimensión disposición para el estudio tiene un nivel de dependencia del $24,1 \%$ de las relaciones en la familia. El resultado es determinante, aproximadamente la cuarta parte de la reacción de indisposición que presentan los estudiantes se debe al clima familiar. Se encuentra que en la pandemia la afectación del clima familiar repercute en la disposición hacia el estudio. Correa (2018) encuentra que existe una correlación altamente significativa entre el funcionamiento familiar y la disposición hacia el estudio de 0,389. Al mismo tiempo Males y Quiroz (2018) encuentran una correlación significativa de 0,194. En la tabla 4 se ve que la relación bidireccional es significativa con un coeficiente de 0,238.

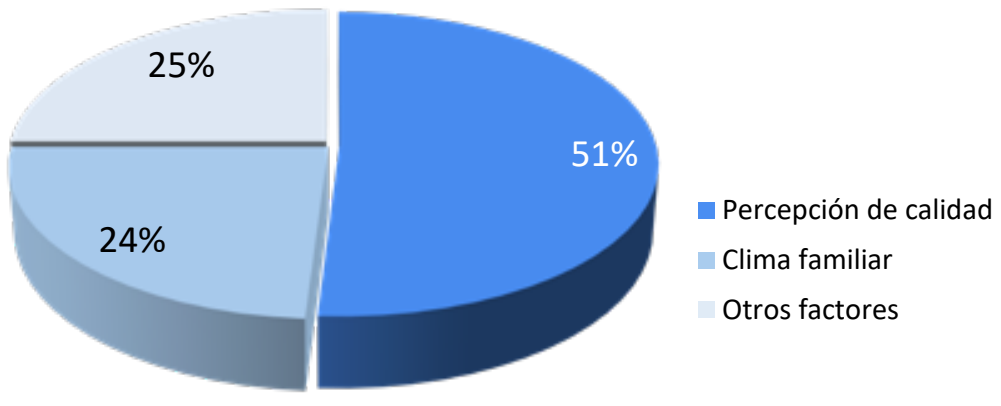

Figura 7. Factores de causa que explican el comportamiento de la variable Disposición Hacia el Estudio

Fuente: Elaboración propia.

Al ser medido el efecto de causa que infringe la dimensión satisfacción del discente hacia la dimensión disposición para el estudio el resultado es contundente, se encuentra que la variable disposición hacia el estudio presenta un nivel de de- 
pendencia del $51 \%$ de la variable satisfacción del discente. El dato que es relevante en este tiempo de pandemia, sugiere que la mayor razón por la que los estudiantes presentan una alta indisposición hacia el estudio es por la percepción de un bajo nivel de calidad del servicio educativo. Se encuentra que en la estrategia educativa m-learning la afectación de los niveles de calidad educativa del sector rural repercuten en la disposición hacia el estudio. En los resultados obtenidos por Fernández (2017) con estudiantes universitarios en Bolivia, encuentra que la satisfacción del estudiante influencia significativamente a la motivación del estudiante y viceversa, con un coeficiente de 0,18 . En la tabla 5 se puede ver que el coeficiente para la relación bilateral es de 0,52. Se observa que para el estudio de Fernández, la variable satisfacción del estudiante se correlaciona muy débilmente con la variable disposición hacia el estudio. El desacuerdo puede corresponder a las diferentes características de la educación básica y media, en comparación con la educación superior. En la educación superior, el estudiante es más consciente de que el nivel de calidad de la educación en gran medida lo pone él mismo.

\section{Conclusiones}

El m-learning lleva el proceso de enseñanza-aprendizaje de la ciencia física con efectividad hasta el lugar donde se encuentran los discentes y contribuye con su posibilidad de aislamiento social a que la enfermedad covid-19 sea contenida. Para la variable disposición hacia el estudio se comprueba que es explicada por el bienestar estudiantil. Al mismo tiempo, habiéndose encontrado que la variable disposición hacia el estudio nos da un referencial para el análisis por su comportamiento inédito, se interpreta que las dos variables del bienestar estudiantil que explican su comportamiento, han sido afectadas de una manera especial también. Se determina que el bienestar estudiantil está siendo afectado en la pandemia con el m-learning. Además, la variable disposición hacia el estudio al estar intrínsecamente relacionada con el aprendizaje, su afectación repercute en los parámetros educativos, lo que sugiere que la educación en el sector rural está involucionando en la pandemia. Se comprueba la Hipótesis del estudio.

A la vista de los resultados obtenidos, se puede decir que, mejorando el clima familiar y aumentando la satisfacción subjetiva por el servicio educativo al aumentar la calidad, se logra que los estudiantes presenten una mayor disposición hacia el estudio, se hace hincapié en que las soluciones se deben dar para las dos dimensiones simultáneamente; ya que, según la dinámica de afectación del bienestar estudiantil, las variables se influyen bilateralmente.

\section{Referencias}

Avena, I y Rivera, D. (2013). Funcionamiento familiar y motivación escolar en alumnos de nivel medio superior. (Tesis de pregrado). Instituto Tecnológico de Sonora, Ciudad de Sonora, Méjico.

Baumert, J., Klieme, E., Neubrand, M., Prenzel, M., Schiefele, U., Schneider, W. y Weiß, M. (1999). Erfassung fächerübergreifender Problemlösekompetenzen in PISA [Elektronische Ressource]. 
Beltrán J. (1993). Procesos, estrategias y técnicas de aprendizaje. Revista Complutense De Educación, 6(2), 235. https://bit.ly/2ZmlYEu

Birenbaum, M. (2002). Evaluación del aprendizaje activo autodirigido en escuelas primarias. Evaluación en educación: principios, política y práctica, 9(1), 119-138.

Buelga, S., Cava, M. y Musitu, G. (2012). Validación de la escala de victimización entre adolescentes a través del teléfono móvil y de internet (CYBVIC). Pan American Journal of Public Health, 32(1), 36-42.

Chiappe, A. y Romero, R. (2018). Condiciones para la implementación del m-learning en educación secundaria: un estudio de caso colombiano. Revista mexicana de investigación educativa, 23(77), 459-481. https://bit.ly/3nurbnp.

Correa A. (2018). Funcionamiento familiar, disposición para el estudio y desempeño académico en Estudiantes de un Instituto de Educación Superior-SENATI 2015. (Tesis de maestría). Universidad Nacional Federico Villarreal, Lima, Perú.

Escobedo, P., Cuervo, M., Sansores, M. y García, J. (2011). Propiedades psicométricas de un instrumento para medir la disposición hacia el estudio. CPU-e, Revista de Investigación Educativa, 12, 73-87.

Fernández, R. (2017). Satisfacción, motivación y rendimiento académico del discente de Educación Secundaria Obligatoria y Bachillerato en la asignatura de Educación Física y con los centros educativos. (Tesis Doctoral). Universidad de Granada, Granada, España. [http:// hdl.handle.net/10481/48345]

Fonseca, A. (2009). El maestro en el contexto de la cultura digital. Magazín Aula Urbana, 74, 4-5.

Gálvez-Nieto, J., Vera-Bachman, D., Cerda, C. y Díaz, R. (2016). Escala de Victimización entre Adolescentes a través del Teléfono Móvil y de Internet: Estudio de validación de una versión abreviada en estudiantes chilenos. Revista Iberoamericana de Diagnóstico y Evaluación-e Avaliação Psicológica, 1(41), 16-27.

Hernández, R., Fernández, C. y Baptista, P. (2010). Metodología de la Investigación. Ciudad de México: Mc Graw Hill.

Katz, R. y Callorda, F. (2013). La banda ancha móvil en la base de la pirámide de América Latina. GSMA Telecom Advisory Services, LLC.

Lugo, M., Ruiz, V., Brito, A. y Brawerman, J. (2016). Revisión comparativa de iniciativas nacionales de aprendizaje móvil en América Latina: Los casos de Colombia, Costa Rica, Perú y Uruguay. UNESCO.

Males V. y Quiroz A._(2018). Influencia del funcionamiento familiar en el desempeño académico de estudiantes de cuarto y quinto año de EGB del colegio "Gálatas" de la ciudad de Quito. (Tesis de pregrado). Universidad Tecnológica Indoamérica, Quito, Ecuador.

Mancinas, A. (2018). Modalidades de cognición en un curso universitario basado en el aprendizaje móvil. Apertura, 10(1), 40-55. https://doi.org/10.32870/ap.v10n1.1159

Moos, R. y Moos, B. (1981). Family Environment Scale. Manual. Palo Alto, CA: Consulting Psychologist Press.

Mendoza, L. (2012). Características de familias de adolescentes desertores escolares matriculados en capacitación laboral. MedULA, 21(1), 47-52.

Nicholls, J., Patashnick, M. y Nolen, S. (1985). Teorías de la educación de los adolescentes. Revista de Psicología de la Educación, 77(6), 683.

Núñez, J. (2009). Motivación, Aprendizaje y rendimiento académico. X Congreso Internacional GalegoPortugües de Psicopedagogía. Braga, Portugal.

Oliva, E. y Pinzón, C. (2012). Medición de la percepción de la calidad del servicio de educación por parte de los estudiantes de la Uptc Duitama. Criterio Libre, 10(16), 159-192.

Ortega, J. y González, D. (2016). El ciberacoso y su relación con el rendimiento académico. Innovación educativa, 16(71), 17-38. https://bit.ly/3pS2Hrd. 
Panimboza, G. (2017). Análisis del rendimiento escolar de los adolescentes del 8vo, 9no, 10mo año para la implementación de un programa en la unidad educativa "John f. Kennedy" de la ciudad de Salinas (Tesis doctoral). Universidad de Guayaquil, Guayaquil, Ecuador.

Paredes, D. (2016). Acoso escolar y clima familiar en estudiantes de $3^{\circ}$ de secundaria de instituciones educativas estatales de la localidad Huaycan - Horacio Zevallos del distrito de Ate (Tesis de maestría). Universidad Nacional Mayor de San Marcos, Lima, Perú.

Ruiz, C. y Guerra, E. (1993). Escala del Clima Social en la Familia FES. Estandarización.

Ruiz, E. y Martínez N. (2012). El aprendizaje colaborativo "escenario para la construcción social de aprendizajes significativos". Cognición, 38.

Sanchez, S. (2015). Entorno familiar y rendimiento académico (Vol. 6). 3Ciencias.

UNESCO (2013). Directrices para las políticas de aprendizaje móvil. París.

UNICEF (2011). Estado mundial de la infancia: La adolescencia, una época de oportunidades. https:// www.unicef.org/spanish/sowc2011/

UNICEF.(2016). Ending the torment: tackling bullying from the schoolyard to cyberspace. New York: United Nations. https://bit.ly/3vJ1gfk

Zapata (s.f.). WOOC: Sesión de aprendizaje con el empleo de WhatsApp. https://bit.ly/3GpcSsQ

\section{Cómo citar en APA:}

Rodríguez-Sarmiento, C. E. (2021). La educación cientifica rural en la modalidad m-learning y su afectación en la pandemia de la covid-19. Revista Iberoamericana de Educación, 87(2), 103-122. https://doi.org/10.35362/rie8724573 